Cell-Free Microfluidic

Determination of P-glycoprotein

Interactions with Substrates and

Inhibitors

Journal Article

Author(s):

Eyer, Klaus (i); Herger, Michael; Krämer, Stefanie-Dorothea (i); Dittrich, Petra S. (1)

Publication date:

2014-12

Permanent link:

https://doi.org/10.3929/ethz-b-000084911

Rights / license:

In Copyright - Non-Commercial Use Permitted

Originally published in:

Pharmaceutical Research 31(12), https://doi.org/10.1007/s11095-014-1431-2 


\title{
Cell-Free Microfluidic Determination of P-glycoprotein Interactions with Substrates and Inhibitors
}

\author{
Klaus Eyer • Michael Herger • Stefanie D. Krämer • Petra S. Dittrich
}

Received: 12 December 2013 / Accepted: 3 June 2014 / Published online: 14 June 2014

(C) Springer Science+Business Media New York 2014

\begin{abstract}
The membrane protein P-glycoprotein (P-gp) plays key roles in the oral bioavailability of drugs, their blood brain barrier passage as well as in multidrug resistance. For new drug candidates it is mandatory to study their interaction with P-gp, according to FDA and EMA regulations. The vast majority of these tests are performed using confluent cell layers of P-gp overexpressing cell lines that render these tests laborious. In this study, we introduce a cell-free microfluidic assay for the rapid testing of drug- P-gp interactions. Cell-derived vesicles are prepared from MDCKII-MDRI overexpressing cells and immobilized on the surface of a planar microfluidic device. The drug is delivered continuously to the vesicles and calcein accumulation is monitored by means of a fluorescence assay and total internal reflection fluorescence (TIRF) microscopy. Only small amounts of compounds $(-10 \mu \mid)$ are required in concentrations of 5, 25 and $50 \mu \mathrm{M}$ for a test that provides within 5 min information on the apparent dissociation constant of the drug and P-gp. We tested 10 drugs on-chip, 9 of which are inhibitors or substrates of $\mathrm{P}$ glycoprotein and one negative control. We benchmarked the measured apparent dissociation constants against an alternative assay on a plate reader and reference data from FDA. These comparisons revealed good correlations between the logarithmic apparent dissociation constants $\left(R^{2}=0.95\right.$ with ATPase assay, $R^{2}=0.93$ with FDA data) and show the reliability of the rapid on-chip test. The herein
\end{abstract}

Electronic supplementary material The online version of this article (doi: | 0. I 007/s | | 095-0 | 4- | 43 | -2) contains supplementary material, which is available to authorized users.

K. Eyer $\cdot$ M. Herger $\cdot$ P. S. Dittrich $(\varangle)$

ETH Zurich, Department of Chemistry and Applied Biosciences

Bioanalytics, Laboratory of Organic Chemistry

Wolfgang-Pauli-Str. 10, 8093 Zurich, Switzerland

e-mail: dittrich@org.chem.ethz.ch

S. D. Krämer

ETH Zurich, Department of Chemistry and Applied Biosciences Biopharmacy and Radiopharmaceutical Sciences, Institute of Pharmaceutical Sciences, 8093 Zurich, Switzerland presented assay has an excellent screening window factor ( $Z^{\prime}$-factor) of 0.8 , and is suitable for high-throughput testing.

KEY WORDS liposome · MDRI · microfluidics ·

P-glycoprotein · TIRF microscopy

\section{INTRODUCTION}

P-glycoprotein (P-gp; MDR1; ABCB1) is a member of the ATP-binding cassette family of transporters with highest expression in the intestine, liver, kidney and blood brain barrier (1,2). P-gp actively transports endogenous substrates such as steroids and cytokines out of the cell (3) and reduces uptake of xenobiotics into the cell. The transport function requires energy from ATP hydrolysis whereby ATP consumption is also significant in the absence of known substrates. The protein recognizes a broad structural variety of compounds with generally hydrophobic and basic properties (4) and is involved in numerous drug-drug interactions (5). It has first been identified as a key player in multidrug resistance of cancer cells $(6,7)$. P-gp plays a major role in the pharmacokinetics of many drugs and drug candidates and is directly or indirectly involved in reducing absorption, restricting distribution and increasing excretion of its substrates. The United States Food and Drug Administration (FDA) and the European Medicines Agency (EMA) both request the evaluation of drug candidates regarding their Pgp transport and inhibition and possible drug-drug interactions related to P-gp transport (8).

Several methods are available to test drug- P-gp interactions in vitro, however, the results are often inconsistent (9). The most widespread assay for in vitro substrate testing for P-gp uses confluent cell layers grown on a porous membrane. The concentration of test compounds is measured on one or both sides of the barrier to evaluate P-gp transport or inhibition. Other approaches make use of competing cell efflux of a 
fluorescent substrate such as daunomycin, doxorubicin or rhodamine 123. The calcein AM assay offers an elegant way to assess P-gp activity (10). Cells are incubated with the nonfluorescent P-gp substrate calcein AM and accumulation of the fluorescent calcein, which is the non-permeating product of enzymatic hydrolysis, is determined. P-gp transport reduces calcein accumulation. These methods usually involve living cells and analysis by fluorescence microscopy (10-12), fluorescence spectrophotometry $(13,14)$, fluorescence activated cell sorting (15), or combinations thereof (16). To overcome difficulties associated with cell-based methods, alternative approaches with artificial membranes were introduced. For example, reconstituted P-gp in membranes or P-gp containing membrane rafts were used to study the potential of a test compound to alter the ATP consumption of P-gp $(17,18)$. Recently, Sasaki et al. (19) succeeded to assess P-gp inhibition in single, inside-out proteoliposomes consisting of membranes of P-gp transfected Sf9 insect cells. The proteoliposomes were immobilized in a microfluidic device using DNA duplexes, and the transport of a fluorescent substrate (rhodamine 123) into individual vesicles was observed in the presence of inhibitors after an incubation time of $20 \mathrm{~min}$. Another rapid method with reconstituted P-gp into liposomes was presented by Melchior et al. (20). However, reconstitution of P-gp suffers a major drawback since it has been shown that the lipid environment influences the activity of P-gp (21-23), and therefore a natural lipid environment would provide, in our opinion, more reliable results. This is further supported by a study that reports synergistic binding effects of lipids, nucleotides and drugs to P-gp (24).

In this study, we introduce a new method for rapid determination of drug- P-gp interactions. We use cell-derived vesicles obtained from human P-gp overexpressing canine kidney epithelial cells (MDCK) so that the natural lipid environment of P-gp is conserved. Unlike cells, these vesicles do not require cell-culture conditions and are not prone to processes involved in apoptosis or cell death when exposed to cytotoxic compounds. We show that these vesicles can be conveniently prepared in large batches and stored for at least 8 weeks without significant loss of activity. The vesicles are immobilized on the surface of a microfluidic channel via a cholesterol linker (25) and exposed to small volumes of test compound solution (Fig. 1, SI Figure 1). Estimation of the apparent transporter-drug dissociation constant $(K)$ is possible within the short time of $5 \mathrm{~min}$ by quantifying the efflux of calcein $\mathrm{AM}$ in the presence and absence of test compound.

\section{MATERIALS AND METHODS}

\section{Materials}

SU-8 and developers for the resists were purchased from Microchem (Newton, MA, USA). AZ-9260 and AZ-400K developer concentrate were obtained from Microchemicals (Ulm, Germany). $1 \mathrm{H}, 1 \mathrm{H}, 2 \mathrm{H}, 2 \mathrm{H}$-perfluorodecyldimethylchlorosilane was obtained from ABCR (Karlsruhe, Germany), and poly(dimethylsiloxane) (PDMS, Sylgard 184) from Dow Corning (Midland, MI, USA). Streptavidin-Texas Red conjugate, calcein AM, Dulbecco's Modified Eagle Medium (DMEM), geneticin sulfate (G418), Glutamax, Hank's buffer salt solution (HBSS), Pen/Strep, fetal calf serum (FCS), phosphate buffered saline (PBS) and Trypsin-EDTA were all obtained from Life Technologies Europe (Zug, Switzerland). Avidin and phosphoenolpyruvate (PEP) were obtained from AppliChem (Gatersleben, Germany). Hexamethyldisilazane was obtained from MERCK (Zug, Switzerland). Adenosine triphosphate (ATP), bovine serum albumin (BSA), biotinylated BSA (bBSA), cytochalasin B, cyclosporine A, elacridar, ergotamine tartrate, lactate dehydrogenase (LD), loperamide hydrochloride $(\mathrm{HCl})$, beta-nicotinamide adenine dinucleotide reduced dipotassium salt $(\mathrm{NADH})$, propranolol $\mathrm{HCl}$, pyruvate kinase $(\mathrm{PK})$, quinidine $\mathrm{HCl}$, ritonavir $\mathrm{HCl}$, reserpin $\mathrm{HCl}$ and verapamil $\mathrm{HCl}$ were all obtained from Sigma-Aldrich (Buchs, Switzerland). Dimethylsulfoxide (DMSO) was from Chemie Brunschwig AG (Basel, Switzerland). Erythromycin, ethylenediaminetetraacetic acid (EDTA), hydrochloric acid 37\%, sucrose and Triton X-100 were obtained from Fluka. Coomassie Brilliant Blue G-250, tris(hydroxymethyl) aminomethane (TRIS) and Tween 20 were obtained from BIORAD (Richmond, CA, USA). Cholesterol-PEG-biotin linker $\left(\mathrm{M}_{\mathrm{w}} 3,400 \mathrm{Da}\right)$ was obtained from Nanocs Inc. (Boston, MA, USA).

\section{Master and Chip Fabrication}

For the fabrication of the master mold and the microfluidic chips, as well as the immobilization protocol, please refer to the Supporting Information. The microfluidic device is shown in SI Figure 1.

\section{Raft Preparation and ATPase Assay}

MDCKII-MDR1 cells, provided by the Netherlands Cancer Institute, were cultured as described in reference (26). Raft extraction and ATPase assay were performed following a slightly modified protocol from Bucher et al. (18). Due to the higher expression levels of P-gp in MDCK-MDR1 cells than in $\mathrm{P} 388 / \mathrm{ADR}$, rafts corresponding to $1 \mu \mathrm{g}$ protein per well were used instead of $5 \mu \mathrm{g}$.

In this assay, ATP hydrolysis results in equimolar NADH oxidation via an enzymatic cascade. ATPase activity was quantified from the decrease in NADH fluorescence with a Synergy HT plate reader (BioTek, Winooski, VT) (18). For data analysis, blank samples served as a background, reflecting the decrease in NADH fluorescence in the presence of rafts but without addition of ATP and drug ( $v_{\text {blank, spontaneous }}$ 
a

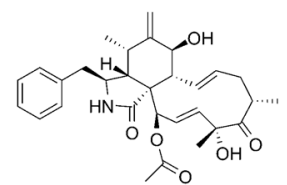

\section{2}

cytochalasin b $\quad$ Incubation

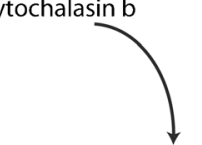

1 cubation

$\left(2^{2}\right)$
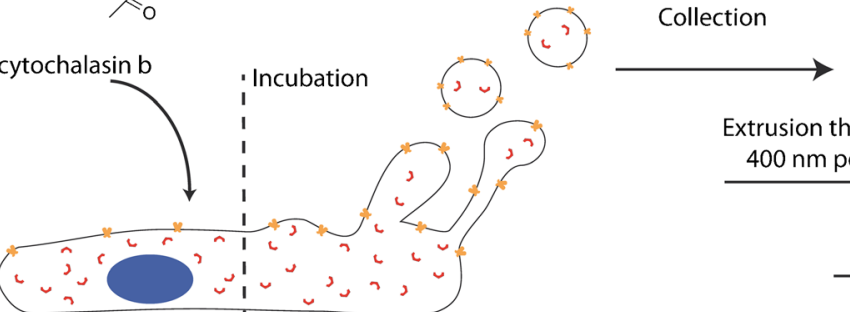

r, 2,
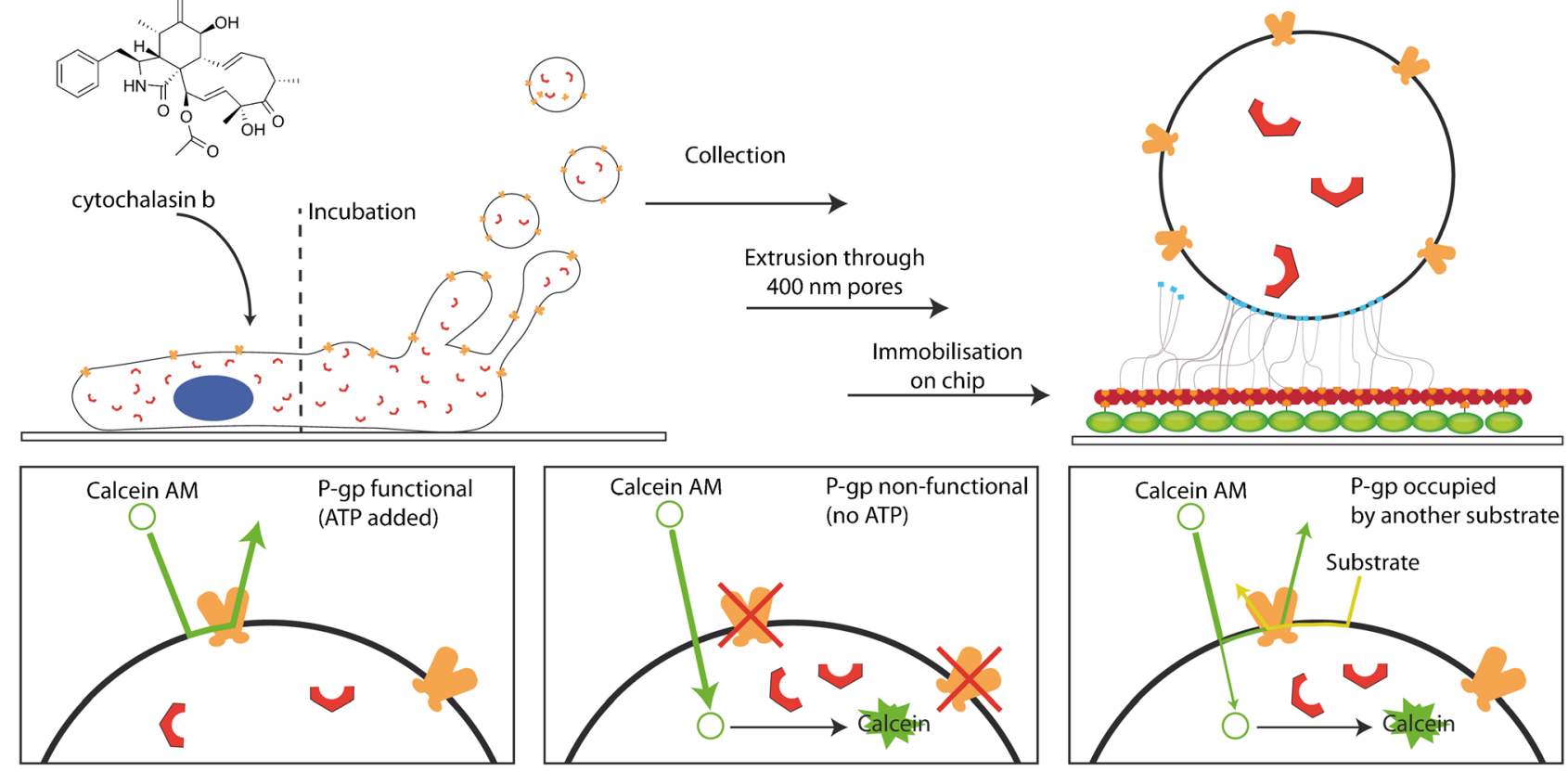

\section{b}
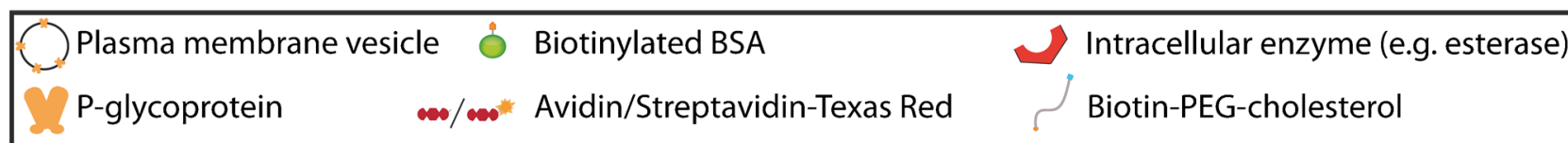

Fig. I Scheme of the method and the assay. (a) Vesicle preparation. Living cells are incubated with cytochalasin B, resulting in the budding of vesicles from the cell membrane and encapsulation of intracellular compounds. Important for the fluorescence assay is the encapsulation of the esterases. The vesicles are collected, extruded and subsequently immobilized on the microfluidic chip via a cholesterol-biotin linker. (b) Fluorescence assay. Left: Addition of calcein AM and ATP to the cell-derived vesicles. P-gp hinders the permeation of the substrate calcein AM. Under these conditions, no fluorescent calcein is formed in the vesicle. Middle: Addition of calcein AM without ATP results in permeation of calcein AM due to non-functional P-gp. This further leads to the formation of fluorescent calcein inside the vesicles. Right: Addition of calcein AM, ATP and an additional P-gp substrate. Here, calcein AM partially permeates the membrane, and is partially effluxed by Pgp. The increase of fluorescent calcein inside the vesicle depends on the extent of drug- P-gp interaction.

oxidation and bleaching of NADH in fluorescence units per time interval). The basal ATPase activity $(v(x=0))$ was determined after ATP addition but in the absence of drug, where $\mathrm{x}$ is the drug concentration. For data analysis, fluorescence decrease was fitted with a linear regression model between 1 and $2 \mathrm{~h}$ after starting the reaction (SI Figure 2a). The obtained slopes $(v(x))$ were first background corrected by subtraction of the slopes from blank samples, and further normalized to the basal activity (Eq. 1). The resulting data was fitted as a function of the drug concentration to a transformation of the Michaelis-Menten Eq. (2):

$v_{a p p}=\left(v(x)-v_{\text {blank }}\right) /\left(v(x=0)-v_{\text {blank }}\right)$

$v_{a p p}=C+v^{\prime} /(1+K / x)$

where $v^{\prime}$ denotes the hypothetical maximal relative druginduced increase in ATP hydrolysis (assuming no inhibition at high concentrations, see below) and $K$ is the apparent dissociation constant (i.e. the drug concentration at half maximal increase in ATP hydrolysis). The relative basal activity $C$ was set to $1, v^{\prime}$ and $K$ were fitted with Eq. 2 and $K$ was used for further analyses. For drugs with a bell-shaped $v_{a p p}-x$ profile (see SI Figure 3 for examples), concentrations above maximal $v_{a p p}$ were excluded from the fit.

\section{Membrane Vesicle Preparation}

The membrane vesicles were prepared as previously described by Pick et al. (27). For vesiculation, MDCKII-MDR1 cells were grown to about $80 \%$ confluence. The cell layer was washed with serum-free RPMI and $10 \mu \mathrm{g} / \mathrm{ml}$ cytochalasin B in serum-free RPMI was added to induce vesiculation. After 15 min incubation at $37^{\circ} \mathrm{C}$, the flask was tapped and the supernatant was collected. The supernatant, containing the shed vesicles, was subsequently extruded 11 times (MicroExtruder, Avanti Polar Lipids, Alabaster AL) through a polycarbonate membrane with $400 \mathrm{~nm}$ pore size (Whatmann Nucleopore, UK). The average diameter of the vesicles was estimated before and after extrusion by dynamic light scattering (DLS; Zetasizer 3000 HAS, Malvern Instruments, UK). 
Total protein amount of three individual vesicle preparations (25 $\mathrm{cm}^{2}$ flasks, $1 \mathrm{ml}$ vesicle preparation) was found to be 9.1 , 6.7 and $8.8 \mu \mathrm{g}$ BSA equivalent respectively, measured by Bradford assay.

\section{Calcein AM Plate Reader Assay to Optimize Vesicle Storage Conditions}

A fluorescence assay was performed to test the P-gp activity of cell-derived vesicles after storage. Fresh and stored vesicle suspensions, respectively, were diluted 1:250 in assay buffer (HBSS with $30 \mathrm{mM}$ Tris $\mathrm{HCl}$ and $0.1 \%(\mathrm{w} / \mathrm{v}) \mathrm{BSA}$ ) and $10 \mu \mathrm{l}$ of the dilutions were pipetted into a 96 well plate and further diluted with $70 \mu \mathrm{l}$ assay buffer. ATP was added in different concentrations in $20 \mu \mathrm{l}$ assay buffer and plate reader measurements were started immediately after addition of $100 \mu \mathrm{l}$ of $2 \mu \mathrm{M}$ calcein AM in assay buffer (final concentration $1 \mu \mathrm{M}$ ) in a Synergy HT plate reader (BioTek, Winooski, VT) for two hours at $37^{\circ} \mathrm{C}$. The excitation and emission wavelengths were 485 and $525 \mathrm{~nm}$, respectively. Duplicates were measured for each storage condition.

Fluorescence increase over time $(v(x))$, i.e., calcein accumulation within the vesicles at different ATP concentrations $x$, were fitted linearly between 10 and $20 \mathrm{~min}$, and data was normalized to the slope without added $\operatorname{ATP}(v(x=0)$, no efflux of calcein AM by P-gp) of each preparation and day (Eq. 3, SI Figure 2b).

$v_{a p p}=v(x) / v(x=0)$

Equation (2) was used to fit the data, with $C=1$. Here, v' denotes the calcein accumulation at infinite ATP concentration $(\mathrm{x} \rightarrow \infty)$ and was used for comparisons of the preparations. Values of $v^{\prime}<1$ indicate functional P-gp while $v^{\prime}$ is 1 in the absence of P-gp transport.

\section{On-Chip Calcein AM Assay}

For TIRF experiments, we mounted the chip on the stage of an inverted microscope (DMI6000B, Leica Microsystems, Switzerland) equipped with a $100 \times$ oil immersion objective (NA 1.47, HCX Plan Apo, Leica Microsystems, Switzerland). The control layer was connected to the nitrogen gas houseline using a custom-built pressure control system and pressurized up to 3 bar to close the valves. The fluid layer was connected to a commercial pressure control system (Fluigent, Paris, France) via Teflon tubing and filled with 10-20 $\mu$ l buffered solution or vesicle suspension.

One of the four inlets was filled with a solution containing $2 \mu \mathrm{M}$ calcein AM in Tris $150 \mathrm{mM} \mathrm{pH} \mathrm{7.4.} \mathrm{This} \mathrm{line} \mathrm{served} \mathrm{as}$ a standard for normalization, as well as positive control for successful surface modification. The other three tubings and inlets were filled with a solution containing $2 \mu \mathrm{M}$ calcein $\mathrm{AM}$, $1 \mathrm{mM}$ ATP and the various drug concentrations in $150 \mathrm{mM}$ Tris pH 7.4.

Initial alignment of the microchip was done with a $561 \mathrm{~nm}$ laser. For measurements, the $488 \mathrm{~nm}$ laser line was used at a low laser power $(15 \%$ power according to the Leica LAS AF control software). The four channels were flushed simultaneously by opening the valves (total flow rate of about $1.5 \mu \mathrm{l} / \mathrm{min}$ ). Image recording was started directly after opening the valves. The fluorescence emitted from calcein inside the native vesicles was recorded using a front-illuminated EMCCD camera (iXon Andor, Belfast, UK) with an exposure time of $400 \mathrm{~ms}$ and a gain of 100 . Images were taken every $10 \mathrm{~s}$ for $5 \mathrm{~min}$.

The image processing software Image J (28) was used to determine the fluorescence intensity within one of the four defined areas just after the four inlet channels were merging together (see Fig. 3a, SI Figure 1a and b). This allowed analysis of four conditions, e.g., four drug concentrations in parallel under otherwise identical conditions. The data from the first 6 frames was discarded to account for the time that is required to flush the test solutions to the observation area. The slope from the calcein AM standard line $(v(x=0))$ served as normalization to reduce chip-to-chip variability (Eq. 4, SI Figure 2c). Here, $x$ denotes the drug concentration in the different solutions.

$v_{a p p}=v(x) / v(x=0)$

In analogy to the calcein AM assay to optimize storage conditions, fluorescence increase is inversely linked to P-gp inhibition (Fig. 1b), i.e. a fully functional P-gp would result in no fluorescence increase, whereas an inhibited transport function would result in a characteristic increase in fluorescence. In this context, $v_{\mathrm{app}}=0$ (no fluorescence increase) would refer to a fully functional, non-inhibited P-gp, whereas $v_{\text {app }}=1$ (maximal fluorescence increase) would refer to a fully inhibited P-gp.

To obtain the apparent dissociation constant $K$, the resulting normalized slopes $v_{a p p}$ were plotted against the drug concentration and the curves were fitted according to Eq. 2. In this assay, $C$ (here 0.02) is the minimal increase in the absence of drug but in the presence of calcein AM and ATP, and $v$ ' the maximal increase at infinite drug concentration $\left(v^{\prime}=1\right)$.

\section{RESULTS}

The principle of our new cell-free assay to study drug- P-gp interactions is shown in Fig. 1. The assay relies on the wellcharacterized interaction between the substrate calcein AM and P-gp $(9,13,15,29)$. In the following, we describe the 
characterization of the cell-derived vesicles, the procedure of the microfluidic assay, and the comparison of the data with an alternative assay that measures ATP hydrolysis and with reference data provided by the FDA.

\section{Vesicle Characterization}

Cell-derived vesicles were prepared according to existing protocols $(27,30)$ and as described in the Materials and Methods section. The exposure of the cells to cytochalasin B that interacts with the actin filaments resulted in budding of the plasma membrane (Fig. 1a). Average diameters of the shed vesicles ranged from 600 to $1,000 \mathrm{~nm}$, in good agreement with former studies (27). Extrusion reduced the average diameter to $150-230 \mathrm{~nm}$ with a polydispersity index between 0.5 and 0.7. This size is ideal for observations with a TIRF microscope. Figure 2a shows the dose-response curve of calcein fluorescence increase for the well-characterized P-gp substrate verapamil, confirming that both $\mathrm{P}$-gp and esterases are active after vesicle preparation and extrusion.

To confirm that P-gp transport rather than calcein AM hydrolysis is rate-limiting in the assay, calcein fluorescence was measured at various ATP concentrations in a plate reader format. The results are shown in Fig. 2c. They not only confirm the presence of active P-gp and of intravesicular esterases, but also indicate that transport by P-gp is ratelimiting as fluorescence was strongly dependent on the ATP concentration. Fluorescence changes were ATP-independent after lysis of the vesicles.

\section{Influence of Storage Conditions on Vesicle Size and Activity ria a Plate Reader Assay}

One advantage of using vesicles compared to cells is the possibility to freeze vesicles for storage with minimum loss of activity (27). We tested various storage conditions (Fig. 2b). After thawing, we determined the diameter of the vesicles by DLS. As shown in Fig. 2b, the vesicle size distribution did not significantly change after one day storage under four of the five tested conditions, including shock freezing in liquid nitrogen after addition of $10 \%$ DMSO and storage at $-80^{\circ} \mathrm{C}$. Shock freezing in the absence of DMSO resulted in broadening of the size distribution. DLS results of the single batches did not significantly change after 2, 4 and 8 weeks storage (data not shown).

The integrity of the vesicles and activity of P-gp and esterases were tested with the calcein AM assay and compared to the results shown in Fig. 2c for the fresh vesicles and for lysed vesicles. The value of $v$ ' was 0 for the freshly prepared vesicles (Eq. 2), i.e. there was no fluorescence increase at high (infinite) ATP concentration. If the vesicles were lysed, the fluorescence increase was independent of the concentration of added ATP and after normalization $v^{\prime}$ was approximately 1 . Therefore, a value close to 0 for $v^{\prime}$ reflects a good freezing protocol with low loss in P-gp activity.

Figure $2 \mathrm{~d}$ shows the fitted values for $v$ ' for all the preparations tested. Interestingly, preparations 2 and 5 lacking DMSO as cryoprotectant showed negative v' values. This may indicate that the vesicles are leaky and ATP can freely diffuse over the membrane, i.e. resulting in a higher intravesicular ATP concentration. Preparations including DMSO (1, 3 and 4) were less affected by the freezing. For the following experiments, we used freezing in liquid nitrogen with added $10 \%$ DMSO and subsequent storage at $-20^{\circ} \mathrm{C}$ (preparation 4) that best preserved the P-gp activity.

\section{Validation of the Microfluidic Calcein AM Assay}

The cell-derived vesicles were immobilized by adsorbed biotinylated BSA, an avidin linker and a cholesterol-PEG-biotin moiety $\left(\mathbf{M}_{\mathrm{w}}\right.$ 3,400 Da, Nanocs Inc.), which tethers the vesicles without disturbing the domain of the membrane facing towards the fluid (25,31). Next, all valves of the device were closed and the test solutions were added to the respective fluidic channels. In the current microchip design, up to 4 different solutions can be introduced and measured at the same time (Fig. 3a, see also SI Figure 1a and b). In our experiments, we used 3 inlets for the introduction of test solutions (different drugs or different concentrations), and one was used for the introduction of the internal standard (here $2 \mu \mathrm{M}$ calcein AM in the absence of both drug and ATP). The standard gives the maximum fluorescence increase and was used for normalization of the signals.

Figure $3 \mathrm{c}$ shows the raw data traces for an experiment with two different concentrations $(25$ and $50 \mu \mathrm{M})$ of verapamil at $1 \mathrm{mM}$ ATP. With no verapamil present, no increase in fluorescence was observed. Addition of verapamil resulted in increasing fluorescence intensity over time, depending on the concentration. For further data analysis, the linearly fitted slopes were normalized to the internal standard that contained neither drug nor ATP (Eq. 4), and showed the maximum increase in fluorescence due to non-hindered permeation of calcein AM.

Several controls were performed to assure the data quality and assay conditions. To optimize the ATP concentration, we investigated the influence of the added ATP concentration on the amount of permeating calcein AM (Fig. 3b). Formation of calcein was inversely correlated to ATP concentration, showing that also on-chip P-gp transport was controlling the kinetics of calcein accumulation. For all further microchip experiments, a concentration of $1 \mathrm{mM}$ ATP was chosen.

Next, we determined the spontaneous, enzymeindependent hydrolysis rate of the calcein $\mathrm{AM}$ ester in buffer with and without added drug. At 60 min after the preparation of the calcein $\mathrm{AM}$ solution, $0.8 \%$ of the 
a

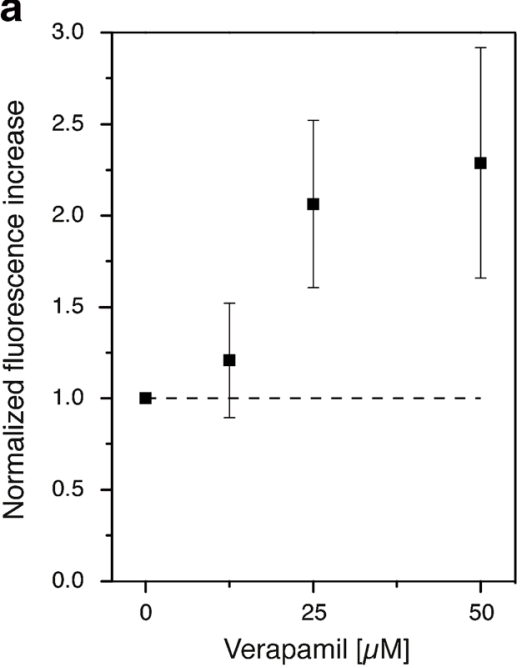

C

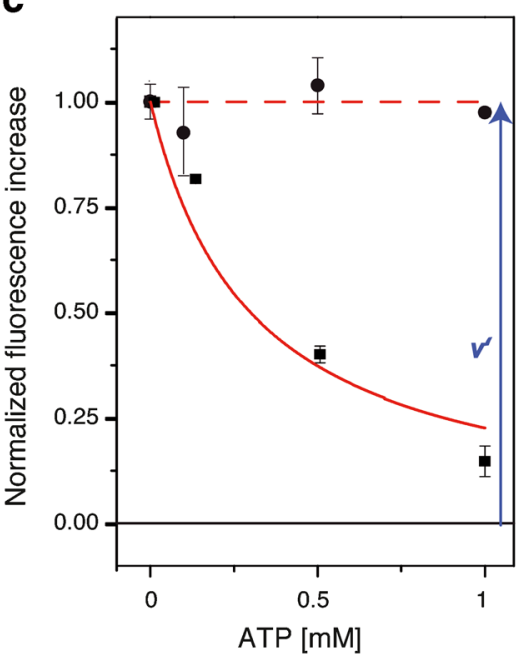

b

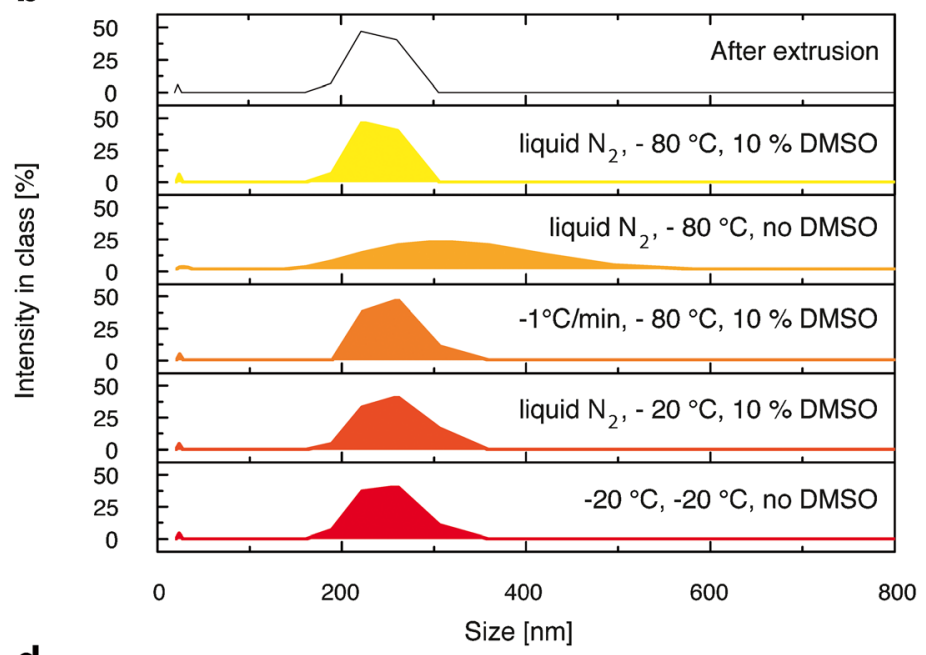

d

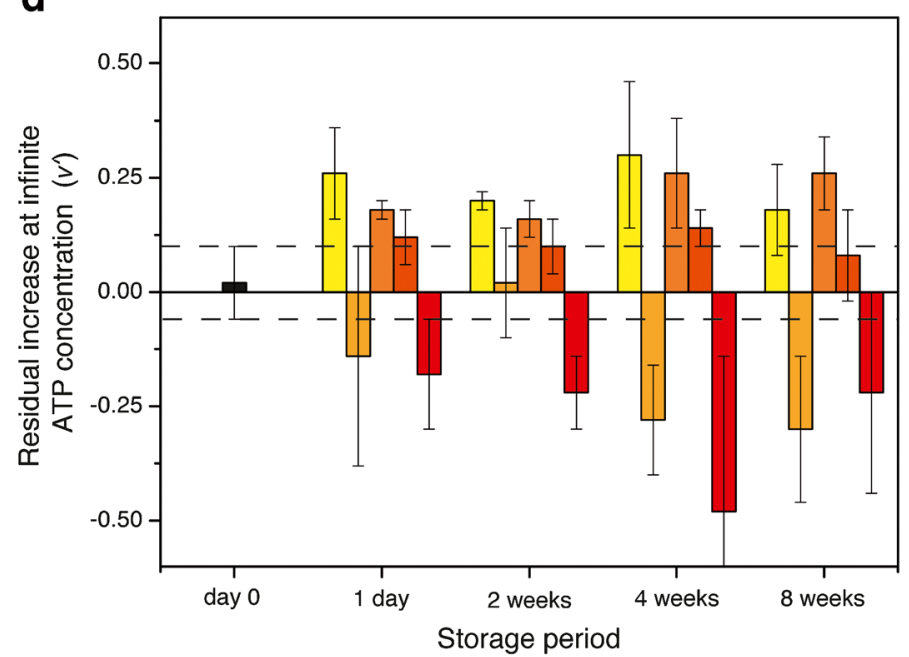

Fig. 2 Vesicle characterization. (a) Dose-response curve of fresh cell-derived vesicles depending on verapamil concentration measured with a plate reader. Addition of more verapamil results in a higher increase of fluorescence, due to the less efficient efflux of calcein AM. (b) Size distribution of the vesicles after extrusion and after one day of storage, measured by dynamic light scattering. Indicated are the freezing procedure, storage temperature and additives. (c) The fluorescence increase of calcein depends on the amount of added ATP (squares). Measurements were conducted using a plate reader. If P-gp or the esterases are non-functional after freezing and thawing or the vesicles are ruptured, the ATP dependency of the fluorescent increase (i.e. the calcein AM transport) is partially or fully lost. This is shown for lysed vesicles (circles). The data was fitted with Eq. 2 to extract $v$ ' (blue arrow) for all preparation conditions, which equals the remaining fluorescence increase at an infinite ATP concentration. Fully functional vesicles have a value of $v^{\prime}=0$; i.e. no fluorescence increase at infinite ATP concentrations, and deviations indicate deterioration. (d) Comparison of the different preparations after various storage times, measured with a plate reader. The vesicles are evaluated by means of the ATP-dependent calcein AM transport depicted in (c). Dashed lines show the standard deviation of freshly prepared vesicles. Colors refer to the same preparations as in (b).

ester was hydrolyzed, independent of the absence or presence of drug (data not shown). Therefore, we assume that enzyme-independent hydrolysis is negligible for the microchip experiments.

Additionally, we evaluated the influence of the flow rate on the signal increase. According to Glaser (32), who modeled the binding kinetics of a ligand to an immobilized antibody inside a flow channel, the relationship between flow rate and signal should be linear in a mass-transfer limited system. Indeed, we found that the fluorescence increase is dependent on the flow rate (Fig. 3d), and we used a constant flow rate of $1.5 \mu \mathrm{l} /$ min in all experiments.
Lastly, we investigated the influence of DMSO on the test system since DMSO is known to enhance the permeation of solutes across the cellular membrane (33). Permeation rates of calcein AM were enhanced at higher concentrations of DMSO (see SI Figure 1c). Therefore, we used a constant concentration of $0.5 \%(\mathrm{v} / \mathrm{v})$ DMSO in all subsequent microfluidic assays.

\section{Determination of Drug- P-gp Interactions with the Microfluidic Assay}

After the control experiments were performed, we tested 9 different substrates/inhibitors of P-gp at various 
Fig. 3 On-chip measurements. (a) Fluorescent micrograph of the microfluidic channel at the measurement region (white square). The four inlets are filled with fluorescein of different concentrations for visualization of the laminar flow regime. (b) ATP dependence of the P-gp transport. Calcein AM can permeate at low ATP concentrations (high fluorescence increase) and its permeation is hindered by P-gp at high ATP concentrations (Compare to Fig. 2c for suspended vesicles). (c) Raw data traces obtained after supply of calcein AM solution with and without ATP and addition of the P-gp substrate verapamil at different concentrations. At time point zero, the valves were opened and the solutions applied to the immobilized vesicles. (d) Flow rate dependency of the fluorescence increase in the vesicles for three conditions. Linear fits are shown as red lines. a

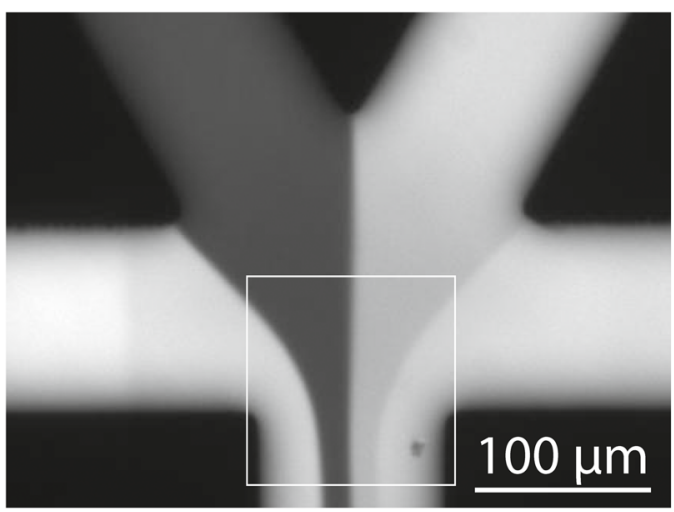

C

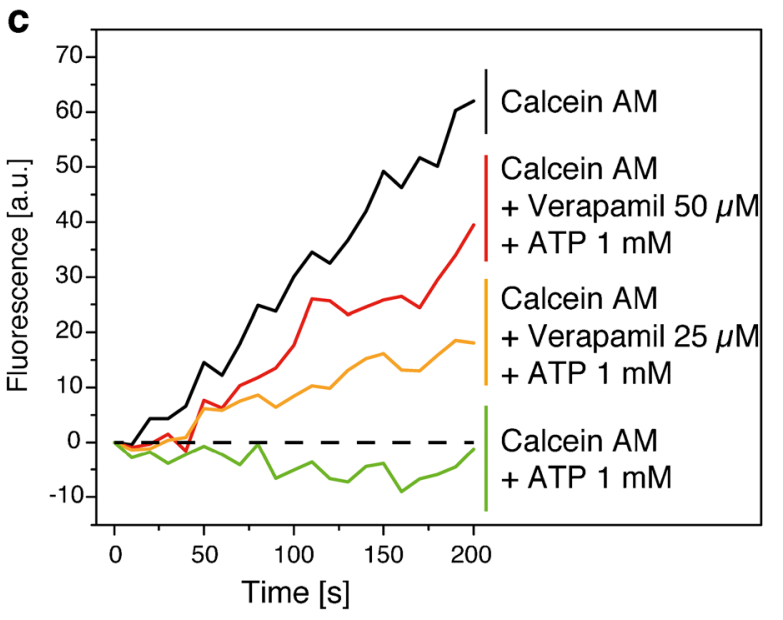

b

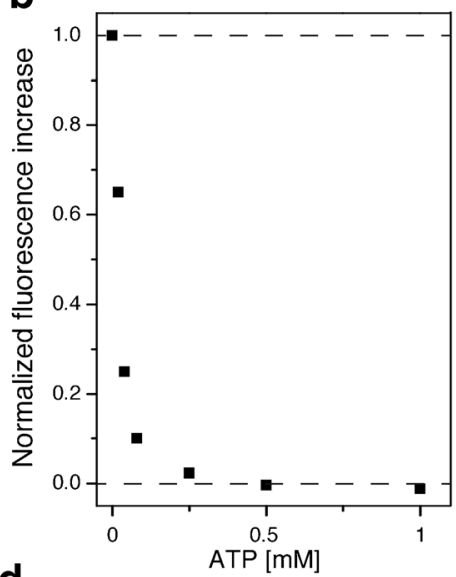

d

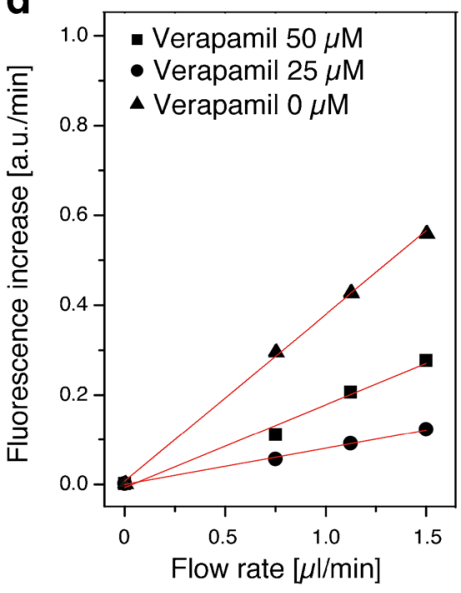

concentrations in the microfluidic calcein AM assay. Each measurement was performed three times (SI Figure 4).

As expected, every substrate or inhibitor that we tested showed an increased fluorescence at higher concentrations. For stronger inhibitors such as cyclosporine A and elacridar, we observed full inhibition at higher concentrations (for values, please refer to SI Table 1), as well as a strong inhibition of P-gp at lower concentrations. When comparing these strong inhibitors with weaker inhibitors such as verapamil or ritonavir, a difference is seen in the profile. Even at the highest concentrations $(50 \mu \mathrm{M})$ weaker inhibitors were not able to fully inhibit P-gp. P-gp substrates such as propranolol or ergotamine showed even less inhibition at the highest concentration, but still a significantly different profile than the negative control. As a negative control we tested geneticin, a hydrophilic basic compound, which shows no interaction with P-gp (34). Indeed, we found very low increases in calcein fluorescence when introducing this substance to the vesicles, presumably resulting from the added DMSO.

From these data sets, we derived the apparent dissociation constants $K$ (Eq. 2, curves and fits are shown in SI Figure 4). The results are shown in Fig. 4.

\section{Determination of Drug- P-gp Interactions with the ATPase Assay}

In parallel to the microfluidic approach, we performed an ATPase assay for comparison. It makes use of the ATPase activity of P-gp (35) that is altered in the presence of drugs interacting with P-gp. In the assay, generated ADP is converted back to ATP in an enzymatic cascade, leading to the conversion of fluorescent NADH to non-fluorescent $\mathrm{NAD}^{+}$ (18). NADH oxidation therefore reflects ATPase activity, which in turn correlates to the transport function.

The measured profile strongly depended on the test substrate (SI Figure 3). Some substrates and inhibitors enhanced ATP hydrolysis (such as quinidine and elacridar), some showed a bell-shaped profile (ergotamine and verapamil), and other drugs showed a decrease in ATPase activity compared to the basal activity (such as cyclosporine A, loperamide, reserpine, erythromycin and ritonavir). These findings are consistent with existing literature $(18,36)$. Decreased activity may result from strong binding of the drug to the P-gp in the raft, resulting in an inhibition of the basal ATPase activity, and/or an inefficient transport of the drug through P-gp. The observation of bell-shaped curves with inhibition at higher concentrations may result from the accumulation of the drug 


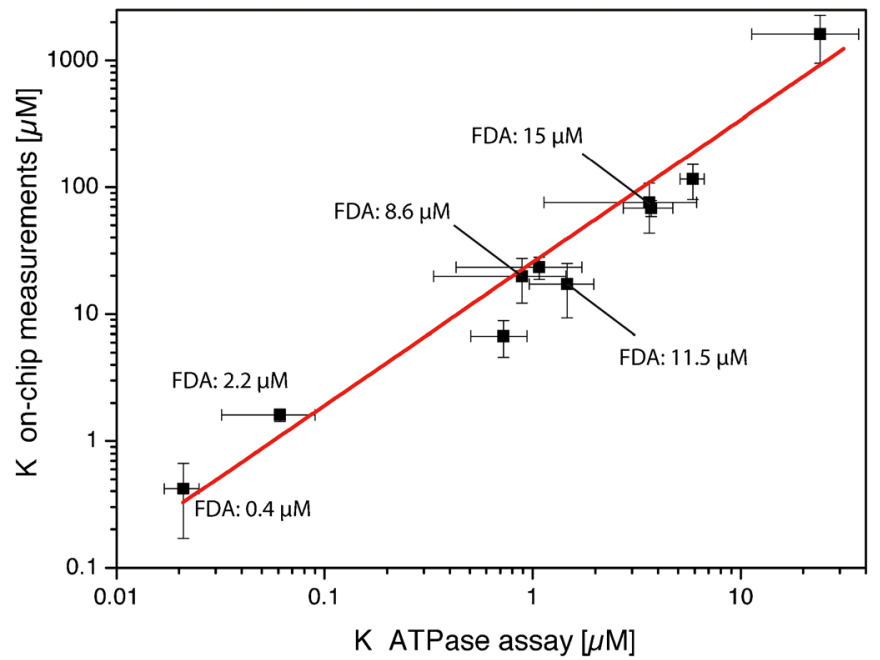

\begin{tabular}{lccc}
\hline Drug & $\begin{array}{c}\text { FDA data } \\
\text { MDCKII-MDR1 } \\
\text { cells }\end{array}$ & $\begin{array}{c}\text { ATPase assay } \\
\text { MDCKII-MDR1 } \\
\text { rafts }\end{array}$ & $\begin{array}{c}\text { On-chip } \\
\text { MDCKII-MDR1 } \\
\text { cell vesicles }\end{array}$ \\
\hline & $K$ & $K$ & $K$ \\
{$[\mu \mathrm{M}]$} & {$[\mu \mathrm{M}]$} & {$[\mu \mathrm{M}]$} \\
\hline Cyclosporine A & 2.2 & $0.061 \pm 0.029$ & $1.61 \pm 0.18$ \\
Elacridar & 0.4 & $0.021 \pm 0.004$ & $0.42 \pm 0.25$ \\
Ergotamine & n.a. & $5.89 \pm 0.77$ & $116.13 \pm 36.20$ \\
Erythromycin & n.a. & $3.64 \pm 2.50$ & $75.88 \pm 32.39$ \\
Loperamide & n.a. & $0.72 \pm 0.22$ & $6.72 \pm 2.13$ \\
Propranolol & n.a. & $24.14 \pm 12.81$ & $1576 \pm 1110$ \\
Quinidine & 8.6 & $0.89 \pm 0.56$ & $19.78 \pm 7.58$ \\
Reserpine & 11.5 & $1.47 \pm 0.50$ & $17.15 \pm 7.82$ \\
Ritonavir & n.a. & $1.08 \pm 0.65$ & $23.36 \pm 4.66$ \\
Verapamil & 15 & $3.71 \pm 1.95$ & $68.54 \pm 9.81$ \\
\hline n.a.: not available & & &
\end{tabular}

Fig. 4 Correlation between $K$ values measured by ATPase assay and fitted values from the on-chip rates. The relation follows a linear trend.

inside the lipid bilayer of the rafts, thereby disrupting the lipid order and inhibiting the functionality of the protein. As negative control, we used geneticin, a drug which does not interact with P-gp (34). As expected, the activity-concentration profile shows a flat line, meaning that ATP hydrolysis was not altered at any of the tested concentrations. The resulting data was fitted with Eq. 2 and the results are shown in Fig. 4.

\section{Comparisons Between FDA, ATPase and on-Chip Data}

Next, we compare the results from the ATPase assay and the on-chip experiments. Figure 4 shows the good linear correlation between $K$ values determined by the microfluidic on-chip approach and obtained with the ATPase assay in the plate reader (Eq. 5, slope $A=19.40 \pm 0.67$, y-intercept $C=0, \mathrm{R}^{2}=$ 0.98). The absolute values of $\mathrm{K}$ differed by a factor of 20 that will be discussed below.

$K_{\text {on-chip }}=A \times K_{\text {ATPase assay }}+C$

Furthermore, the extracted apparent dissociation constants of the microfluidic approach and the ATPase assay show the same general trend of the dissociation constants as the data available from the FDA (37), underlining the reliability of both methods $\left(\mathrm{R}^{2}=0.93\right.$ for on-chip, $\mathrm{R}^{2}=0.95$ for ATPase assay for the logarithmic values).

\section{Fast Assessment of the Apparent Dissociation Constants}

The on-chip system may be suited for high throughput determination of the potency of a drug or drug candidate to interact with P-gp. As shown above, we found a good correlation between the results of the on-chip and ATPase assay (Fig. 4). Comparing the normalized fitted slopes $\left(v_{a p p}\right)$ at a defined concentration of the test compounds versus $\mathrm{K}$ of the ATPase assay in a semi-log plot followed a dose-response like behavior for all three tested concentrations (SI Figure 5a). As expected from the correlation in Fig. 4, the half maximal slope on-chip (meaning the indicated concentration on-chip would equal $\mathrm{K}$ on-chip) corresponded to $\mathrm{K}$ of the ATPase assay at 20-fold lower concentration than on-chip. The fitting parameters of the dose-response curves are shown in SI Table 2.

To demonstrate the use of the system, we tested the model compound doxorubicin at three different concentrations (5, 25 and $50 \mu \mathrm{M}$ ) to determine the apparent dissociation constant of the doxorubicin- P-gp interaction. First, we fitted the on-chip slopes with Eq. 2 (SI Figure 5b). This revealed a $K$ value of $14.40 \pm 5.64 \mu \mathrm{M}$. Next, we used the offline calibration from the ATPase assay for the determination of the respective $K$ in the ATPase assay (SI Figure 5a and fit parameters in SI Table 2). We found a good agreement between the calculated $K$ values for all three different concentrations (SI Figure 5 c, $0.76 \pm 0.31,0.35 \pm 0.01$ and $1.10 \pm 0.04$ for 50,25 and $5 \mu \mathrm{M}$ respectively). The mean apparent dissociation constant of the doxorubicin- P-gp interaction estimated for the ATPase assay according to the correlations in SI Figure 5b was $0.73 \pm$ $0.35 \mu \mathrm{M}$. The advantage of using the ATPase assay for calibration is that a single point determination at a specific concentration is enough to estimate the $\mathrm{K}$ value, therefore allowing much higher throughput.

\section{DISCUSSION}

In our assay, we combine the use of immobilized Pglycoprotein overexpressing cell-derived vesicles with microfluidics. By using cytochalasin B for the production of cell-derived vesicles, only vesicles from the plasma membrane 
are formed, with a high proportion of P-gp in the vesicles. Competing processes involving transporter proteins present in other cell compartments are reduced, so that a high and specific drug- P-gp interaction is obtained. Furthermore, the vesicles contain cytoplasmatic esterases (27), which are required in the assay presented here.

The use of vesicles instead of cells offers several advantages. Vesicles can be produced and stored over longer time periods, retaining their functionality. The monitoring of cell health during the experiment is not required. This is of particular interest when testing cytotoxic compounds. Furthermore, in combination with the microfluidic platform, only small amounts of test compound (10-20 $\mu \mathrm{L}$ in $\mu \mathrm{M}$ concentrations) and vesicle solution are needed. Small sample volumes and the possibility to store the cell-derived vesicles makes it possible to produce a large batch of vesicles once and use it for many experiments, therefore reducing heterogeneities that could occur during cultivation of cells. In contrast to plate reader formats, it is not required to determine the actual concentration of the vesicles, as long as the surface of the device is fully covered by vesicles. Small deviations of the individual vesicle diameter within a preparation, $\mathrm{P}$-gp density, or concentration of the esterases are also not influencing the results as the observation area covers many (ca. 50,000) immobilized vesicles and the fluorescence intensities are therefore averages. Lastly, the natural lipid environment present in the cell-derived vesicles is beneficial for P-gp studies since it mimics physiological conditions. However, small alterations in the cholesterol concentrations are inserted due to the immobilization of the vesicles. Upon immobilization, the increase in cholesterol content in the outer leaflet over the whole vesicle can be estimated to be 0.36 , and locally $4.20 \%$ (see calculation in the SI). We think that these increases are minor deviations from the natural composition, and their influence can be neglected.

By using TIRF microscopy, a gain in sensitivity and specificity is achieved. Due to the measurements on the surface where the liposomes are immobilized, the background fluorescence from the channel is reduced, and only fluorophores inside the vesicles are excited. Optimal is the use of vesicles with sizes that roughly match the height of the evanescent field $(\sim 200 \mathrm{~nm})$.

The combination with microfluidics provides a constant environment due to the flow, i.e. the concentrations of ATP, drug and calcein AM are constant over time and are not varying over the measurement period. This is in contrast to plate reader measurements, where e.g. the decreasing ATP concentration has to be considered in data interpretation or compensated during the measurements. Besides this, the method is also suitable for plate reader measurements at high throughput using a suspension of the cell-derived vesicles or surfaceimmobilized vesicles and a TIRF microscope.
We used a simple, low-cost microfluidic device made of PDMS. Because of this, absorption or diffusion of the typically lipophilic substances into the PDMS have to be considered (38). To minimize this effect, we used short transport channels from the inlet to the actual observation position and the diffusion of molecules from the central channel towards the walls is negligible within the short residence time of the substances ( $300 \mathrm{~ms}$ at low flow rates of $1.5 \mu \mathrm{L} / \mathrm{min}$ ). Additionally, we selectively measured slightly above the channels glass bottom, where no absorption to PDMS is expected. Therefore, the concentration of the drug can be considered constant and equal to the supplied concentration. For more routine applications, PDMS can easily be exchanged with a material that is less absorbent, such as glass.

We have demonstrated the use of the system by successfully correlating the fitted on-chip $K$ values with an ATPase assay and values given by the FDA. While we always found good correlations and matching trends, the absolute value of the apparent dissociation constants differ between the methods. These variances must be caused by the different experimental conditions. For example, the FDA measurements were performed with cell monolayers, the ATPase assay used lipid rafts and the microfluidic device relied on cell-derived vesicles. The deviation from cell-derived vesicles to cells is minor (factor 2), and could be assigned to the difference in fluid handling, different parameters in the assay, and/or interferences with cellular processes that do not occur in the vesicles. The highest deviation was found when comparing the two cell-like systems with the lipid rafts (ATPase assay). Here, other factors could come into play such as the different lipid environment that would influence the partitioning of the drugs and therefore the concentration present at the transporter.

In addition, apparent dissociation constant depends on the concentration of additional endogenous or added substrates, such as calcein AM. In our assays, calcein AM was at $1 \mu \mathrm{M}$, this is about 8-fold higher than its reported Michaelis Menten constant of $0.12 \mu \mathrm{M}$ for P-gp transport (39). The drug concentration at half maximal calcein AM transport is, therefore, about 10-fold higher in our assay than it would be in the absence of calcein AM, in analogy to the Cheng-Prussof equation relating $\mathrm{IC}_{50}$ with absolute $\mathrm{K}_{\mathrm{i}}$ values. This could explain in part the 20-fold higher $\mathrm{K}$ values in the calcein AM than the ATPase assay.

Compounds interacting with P-gp are frequently classified as substrates, modulators or inhibitors. However, the differentiation between these classifications is not clearly defined and not used consistently. Several compounds such as verapamil or loperamide with low dissociation constants are often classified as substrates and inhibitors. In these cases, both the $K$ and the in vivo concentration of the drug must be considered for evaluation of the effect. Figure 5 depicts a graph showing the transport activity of P-gp (i.e. $v_{a p p}$ ) versus the quotient $[\mathrm{drug}] / K$ for all tested compounds and concentrations. Since 
they all follow Michaelis-Menten behavior, the appearance of the curve is the logical consequence of a saturation experiment. As can be seen, the remaining transport activity of P-gp is independent of the compound, but dependent on the ratio of the administered concentration to the dissociation constant. If [drug] $/ K>>1$, the compound is inhibiting P-gp, meaning that there is only small amounts of free P-gp available (i.e. Pgp is mainly bound to the inhibiting compound). As a result of this, a P-gp substrate turns to an inhibitor, where it completely occupies the protein (if supplied in high enough concentrations). The drug can be classified as a substrate if the tested drug concentration is simular to the dissociation constant ([drug] $/ K \approx 1$ ). Furthermore, if [drug] $/ K<<1$, the drug would be classified as a non-substrate, even if there would be an interaction with $\mathrm{P}$-gp observable at higher concentrations. The relationship between [drug], $K$ and lipoidal (passive) permeation is shown in SI Figure 6. The data shows the importance of the concentration used in the assays, and may help to explain why different drug compounds are classified as inhibitors, substrates, and/or non-substrates by different assays performed by different groups.

Our method enables fast evaluation of the interaction of a potential drug molecule with the transport protein P-gp and the here presented assay is, in theory, compatible with highthroughput measurements. An excellent screening window factor (Z'-factor) of 0.8 can be calculated for the assay (40).

To further support this hypothesis, we performed an experiment with another substrate, doxorubicin. The whole onchip measurements took $15 \mathrm{~min}$ (four triplicates each) and used up $50 \mu \mathrm{l}$ of each dilution, demonstrating the rapidness and economic value of the system. After calibration with the ATPase measurements, a single on-chip determination can be enough for rapid, but less accurate, assessment of $K$. Therefore it would be possible to test up to three different substances at once, decreasing the measurement time to $5 \mathrm{~min}$ and increasing the throughput.

We are also confident that the combination of the assay with microfluidics has a great potential for high throughput screening. With modifications of the current chip design additional benefits could be achieved with respect to parallelization and further miniaturization (channels are closed, i.e. no evaporation of the sample). In future, the device design could be modified with a microfluidic concentration gradient generator (41) to introduce many different drug concentrations in parallel and hence, obtain more data points for the dose-response curve. Moreover, different cell-derived vesicles embedding other membrane transporters or receptors of interest could be immobilized at different positions of the channel, so that the interactions of the drug with these vesicles can be assessed on one single platform provided that these interactions produce a fluorescent or bioluminescent readout signal.

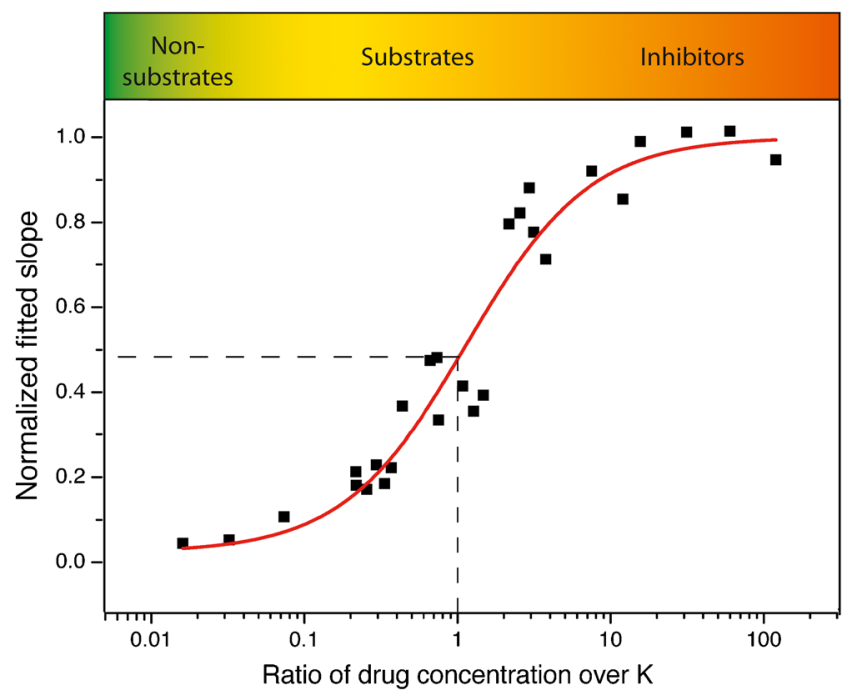

Fig. 5 Transport activity of P-gp obtained on chip (i.e. the normalized rate of fluorescence increase, $v_{\text {app. }}$ ) versus the ratio of drug concentration to the fitted on-chip dissociation constant, $K$. Large ratios indicate an inhibitor-like effect of the drug and smaller ratios indicate that the drug is classified as a substrate. Very small ratios lead to classification as non-substrates.

\section{CONCLUSION}

In conclusion, we have introduced a rapid and economic method for investigations of drug- P-gp interactions. We were able to determine $K$ values for various compounds with our microfluidic system. The results were in good agreement with an off-chip method (ATPase assay) as well as with FDA data. The method allows predicting the type of P-gp interaction of test compounds.

\section{ACKNOWLEDGMENTS AND DISCLOSURES}

We thank Phillip Kuhn for pilot experiments and many helpful discussions. We thank Tom Robinson for careful and thorough proofreading of the manuscript. We also acknowledge the clean room facility FIRST and the LMG (Light Microscopy Centre) at the ETH Zurich, and Pascal Verboket and Phillip Kuhn for the fabrication of the wafers. We are grateful to the financial support of OPO foundation Switzerland (SDK), the European Research Council (ERG Starting Grant, Project No. 203428, nuLIPIDs, PSD) and Merck Serono (PSD, EK).

\section{REFERENCES}

1. Gottesman MM, Pastan I, Ambudkar SV. P-glycoprotein and multidrug resistance. Curr Opin Genet Dev. 1996;6:610-7.

2. Cascorbi I. P-glycoprotein: tissue distribution, substrates, and functional consequences of genetic variations, Handb Exp Pharmacol. $2011 ; 261-283$. 
3. Hartz AMS, Miller DS, Bauer B. Restoring blood-brain barrier Pglycoprotein reduces brain amyloid-beta in a mouse model of Alzheimer's disease. Mol Pharmacol. 2010;77:715-23.

4. Hitchcock SA. Structural modifications that alter the P-glycoprotein efflux properties of compounds. J Med Chem. 2012;55:4877-95.

5. International Transporter Consortium, Giacomini KM, Huang SM, Tweedie DJ, Benet LZ, Brouwer KLR, et al. Membrane transporters in drug development. Nature. 2010;9:215-36.

6. Juliano RL, Ling V. A surface glycoprotein modulating drug permeability in Chinese hamster ovary cell mutants. Biochim Biophys Acta. 1976;455:152-62.

7. Gottesman MM, Fojo T, Bates SE. Multidrug resistance in cancer: role of ATP-dependent transporters. Nat Rev Cancer. 2002;2:4858.

8. Prueksaritanont T, Chu X, Gibson C, Cui D, Yee KL, Ballard J, et al. Drug-drug interaction studies: regulatory guidance and an industry perspective. AAPS J. 2013;15:629-45.

9. Polli JW, Wring SA, Humphreys JE, Huang L, Morgan JB, Webster $\mathrm{LO}$, et al. Rational use of in vitro P-glycoprotein assays in drug discovery. J Pharmacol Exp Ther. 2001;299:620-8.

10. Eneroth A, Åström E, Hoogstraate J, Schrenk D, Conrad S, Kauffmann HM, et al. Evaluation of a vincristine resistant Caco-2 cell line for use in a calcein AM extrusion screening assay for Pglycoprotein interaction. Eur J Pharm Sci. 2001;12:205-14.

11. Nelson EJ, Zinkin NT, Hinkle PM. Fluorescence methods to assess multidrug resistance in individual cells. Cancer Chemoth Pharm. 1998;42:292-9.

12. von Richter O, Glavinas H, Krajcsi P, Liehner S, Siewert B, Zech K. A novel screening strategy to identify $\mathrm{ABCB} 1$ substrates and inhibitors. N-S Arch Pharmacol. 2009;379:1 1-26.

13. Liminga G, Nygren P, Larsson R. Microfluorometric evaluation of calcein acetoxymethyl ester as a probe for P-glycoprotein-mediated resistance: effects of cyclosporin A and its nonimmunosuppressive analogue SDZ PSC 833. Exp Cell Res. 1994;212:291-6.

14. Glavinas H, von Richter O, Vojnits K, Mehn D, Wilhelm I, Nagy T, et al. Calcein assay: a high-throughput method to assess P-gp inhibition. Xenobiotica. 2011;41:712-9.

15. Legrand O, Simonin G, Perrot JY, Zittoun R, Marie JP. Pgp and MRP activities using calcein-AM are prognostic factors in adult acute myeloid leukemia patients. Blood. 1998;91:4480-8.

16. Ansbro MR, Shukla S, Ambudkar SV, Yuspa SH, Li L. Screening compounds with a novel high-throughput ABCB1-mediated efflux assay identifies drugs with known therapeutic targets at risk for multidrug resistance interference. PLoS ONE. 2013;8:e60334.

17. Eytan GD, Regev R, Assaraf YG. Functional reconstitution of Pglycoprotein reveals an apparent near stoichiometric drug transport to ATP hydrolysis. J Biol Chem. 1996;271:3172-8.

18. Bucher K, Besse CA, Kamau SW, Wunderli-Allenspach H, Krämer SD. Isolated rafts from adriamycin-resistant P388 cells contain functional ATPases and provide an easy test system for P-glycoproteinrelated activities. Pharm Res. 2005;22:449-57.

19. Sasaki H, Kawano R, Osaki T, Kamiya K, Takeuchi S. Singlevesicle estimation of ATP-binding cassette transporters in microfluidic channels. Lab Chip. 2012;12:702-4.

20. Melchior DL, Sharom FJ, Evers R, Wright GE, Chu JWK, Wright $\mathrm{SE}$, et al. Determining P-glycoprotein-drug interactions: evaluation of reconstituted P-glycoprotein in a liposomal system and LLC-MDR1 polarized cell monolayers. J Pharmacol Toxicol. 2012;65:64-74.

21. Belli S, Elsener PM, Wunderli-Allenspach H, Krämer SD. Cholesterol-mediated activation of P-glycoprotein: distinct effects on basal and drug-induced ATPase activities. J Pharm Sci. 2009;98:1905-18.
22. Clay AT, Sharom FJ. Lipid bilayer properties control membrane partitioning, binding, and transport of p-glycoprotein substrates. Biochemistry. 2013;52:343-54.

23. Cannon RE, Peart JC, Hawkins BT, Campos CR, Miller DS. Targeting blood-brain barrier sphingolipid signaling reduces basal P-glycoprotein activity and improves drug delivery to the brain. Proc Natl Acad Sci U S A. 2012;109:15930-5.

24. Marcoux J, Wang SC, Politis A, Reading E, Ma J, Biggin PC, et al. Mass spectrometry reveals synergistic effects of nucleotides, lipids, and drugs binding to a multidrug resistance efflux pump. Proc Natl Acad Sci U S A. 2013;1 10:9704-9.

25. Kuhn P, Eyer K, Robinson T, Schmidt FI, Mercer J, Dittrich PS. A facile protocol for the immobilisation of vesicles, virus particles, bacteria, and yeast cells. Integr Biol (Camb). 2012;4:1550-5.

26. Bakos E, Evers R, Szakács G, Tusnády GE, Welker E, Szabó K, et al. Functional multidrug resistance protein (MRP1) lacking the Nterminal transmembrane domain. J Biol Chem. 1998;273:32167-75.

27. Pick H, Schmid EL, Tairi A-P, Ilegems E, Hovius R, Vogel H. Investigating cellular signaling reactions in single attoliter vesicles. J Am Chem Soc. 2005; 127:2908-12.

28. Schneider CA, Rasband WS, Eliceiri KW. NIH Image to ImageJ: 25 years of image analysis. Nat Meth. 2012;9:671-5.

29. Essodaigui M, Broxterman HJ, Garnier-Suillerot A. Kinetic analysis of calcein and calcein-acetoxymethylester efflux mediated by the multidrug resistance protein and P-glycoprotein. Biochemistry. 1998; $37: 2243-50$

30. Mao Z, Cartier R, Hohl A, Farinacci M, Dorhoi A, Nguyen T-L, et al. Cells as factories for humanized encapsulation. Nano Lett. 2011;11:2152-6.

31. Eyer K, Paech F, Schuler F, Kuhn P, Kissner R, Belli S, et al. A lipsomal fluorescence assay to study permeation kinetics of drug-like compounds across lipid membranes. JCR. 2013;173:102-9.

32. Glaser RW. Antigen-antibody binding and mass transport by convection and diffusion to a surface: a two-dimensional computer model of binding and dissociation kinetics. Anal Biochem. 1993;213:152-61.

33. Gurtovenko AA, Anwar J. Modulating the structure and properties of cell membranes: the molecular mechanism of action of dimethyl sulfoxide. J Phys Chem B. 2007;111:10453-60.

34. Mechetner EB, Roninson IB. Efficient inhibition of P-glycoproteinmediated multidrug resistance with a monoclonal antibody. Proc Natl Acad Sci U S A. 1992;89:5824-8.

35. Ambudkar SV, Dey S, Hrycyna CA, Ramachandra M, Pastan I, Gottesman MM. Biochemical, cellular, and pharmacological aspects of the multidrug transporter 1. Annu Rev Pharmacol. 1999;39:361-98.

36. Garrigues A, Nugier J, Orlowski S, Ezan E. A high-throughput screening microplate test for the interaction of drugs with Pglycoprotein. Anal Biochem. 2002;305:106-14.

37. fda.gov, Center for Drug Evaluation and Research, 07.10.2013.

38. Regehr KJ, Domenech M, KoepselJT, Carver KC, Ellison-Zelski SJ, Murphy WL, et al. Biological implications of polydimethylsiloxanebased microfluidic cell culture. Lab Chip. 2009;9:2132-9.

39. Essodaigui M, Broxterman HJ, Garnier-Suillerot A. Kinetic analysis of calcein and calcein-acetoxymethylester efflux mediated by the multidrug resistance protein and P-glycoprotein. Biochemistry. 1998;37:2243-50.

40. Zhang J, Chung T, Oldenburg K. A simple statistical parameter for use in evaluation and validation of high throughput screening assays. J Biomol Screen. 1999;4:67-73.

41. Jeon NL, Dertinger SKW, Ghiu DT, Choi IS, Stroock AD, Whitesides GM. Generation of solution and surface gradients using microfluidic systems. Langmuir. 2000;16:8311-6. 